\title{
Impaired beta cell glucose sensitivity rather than inadequate compensation for insulin resistance is the dominant defect in glucose intolerance
}

\author{
A. Mari • A. Tura • A. Natali • M. Laville • M. Laakso • \\ R. Gabriel • H. Beck-Nielsen • E. Ferrannini • \\ The RISC Investigators
}

Received: 28 September 2009 / Accepted: 1 December 2009/Published online: 9 January 2010

(C) Springer-Verlag 2010

\author{
Abstract \\ Aims/hypothesis It is commonly thought that hyperglycaemia \\ results from insufficient compensation of insulin secretion for \\ insulin resistance. To verify this hypothesis, we assessed beta \\ cell function and insulin sensitivity (IS) in a large cohort of \\ volunteers with normal glucose tolerance (NGT) or impaired \\ glucose regulation (IGR), i.e. impaired glucose tolerance or \\ impaired fasting glucose. \\ Methods In men and women with NGT $(n=1,123)$ or IGR \\ $(n=156)$ (age $44 \pm 8$ years, BMI $25 \pm 4 \mathrm{~kg} / \mathrm{m}^{2}$, mean \pm SD) \\ Electronic supplementary material The online version of this article \\ (doi:10.1007/s00125-009-1647-6) contains supplementary material, \\ which is available to authorised users.

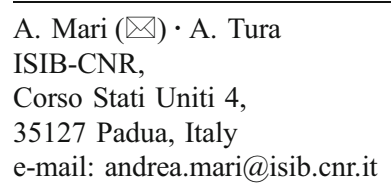 \\ A. Natali $\cdot$ E. Ferrannini \\ Department of Internal Medicine and C.N.R. Institute of Clinical \\ Physiology, University of Pisa School of Medicine, \\ Pisa, Italy \\ M. Laville \\ CRNHRA, Lyon 1 University, \\ Hospices Civils de Lyon, \\ Lyon, France \\ M. Laakso \\ Department of Medicine, University of Kuopio, \\ Kuopio, Finland \\ R. Gabriel \\ Unidad de Investigacion, Hospital Universitario La Paz, \\ Madrid, Spain \\ H. Beck-Nielsen \\ Department of Endocrinology M, Odense University Hospital, \\ Odense, Denmark
}

we measured: (1) IS by clamp; (2) insulin secretion rates (ISR) and beta cell glucose sensitivity (=slope of the insulin secretion/plasma glucose dose-response) by C-peptide deconvolution and OGTT modelling; and (3) acute insulin response to intravenous glucose.

Results After controlling for centre, sex, age and BMI, fasting and total ISR were inversely related to IS in both groups, whereas beta cell glucose sensitivity was not. Acute insulin response was reciprocally related to IS in both groups, but the relationships were incompatible with inadequate compensation and significance was lost after controlling for fasting ISR. In IGR vs NGT, IS was impaired (92 [75] vs 133 [86] $\mu \mathrm{mol} \mathrm{min}{ }^{-1}$ $[\mathrm{kg} \text { fat-free mass }]^{-1}[\mathrm{nmol} / \mathrm{l}]^{-1}$, median [interquartile range], $p<0.0001$ ) as was beta cell glucose sensitivity (69 [46] vs 119 [83] pmol $\left.\mathrm{min}^{-1} \mathrm{~m}^{-2}[\mathrm{nmol} / 1]^{-1}, p<0.0001\right)$, whereas fasting and total ISR were increased (35\% and $25 \%$, respectively, $p<$ $0.0001)$. In fully adjusted models, beta cell glucose sensitivity was the strongest determinant of OGTT glucose levels.

Conclusions/interpretation Insulin resistance normally upregulates the secretory tone, with no evidence of defective compensation in IGR. In contrast, beta cell glucose sensitivity is independent of insulin resistance, but a key determinant of glucose tolerance. This suggests that hyperglycaemia results from an intrinsic beta cell defect rather than from inadequate compensation for insulin resistance.

Keywords Beta cell compensation - Beta cell function · Glucose tolerance · Hyperglycaemia · Insulin sensitivity · Pre-diabetes
Abbreviations
AIR Acute insulin response to intravenous glucose
FFM Fat-free mass
IGR Impaired glucose regulation
IS Insulin sensitivity 
ISR Insulin secretion rates

NGT Normal glucose tolerance

RISC Relationship between insulin sensitivity and cardiovascular disease

\section{Introduction}

There is a wide consensus that insulin resistance and beta cell dysfunction are essential factors in the pathogenesis of type 2 diabetes [1,2]. It is also well established that beta cell function is, at least in some respect, inversely related to insulin sensitivity (IS) [3-6]. Thus, insulin-resistant states are not necessarily associated with glucose intolerance because insulin secretion can be upregulated to cope with the increased demand. This has led to the concept that type 2 diabetes arises from a defect in secretory compensation for insulin resistance. Thus as long as a decrease in IS is matched by a commensurate increase in beta cell function, glucose tolerance is maintained; when beta cell adaptation is insufficient, hyperglycaemia develops. In a broad sense, this is a tautology, for if secretory function were better, glucose tolerance would be maintained. However, in physiological terms inappropriate compensation implies the existence of a specific cellular mechanism, which reacts to insulin resistance and maintains glucose tolerance [7]. The concept of inappropriate compensation in hyperglycaemic states has its roots in studies using intravenous glucose as the physiological test and the acute insulin response to intravenous glucose (AIR) as the index of beta cell response. These studies have proposed a hyperbolic model for the relationship between AIR and IS; in log-log representation, the relationship is a straight line with a slope of -1 and the product of AIR and IS is called the disposition index [4]. In a recent study using this paradigm [8], the hyperbolic relationship was reported to be shifted towards the origin in glucose-intolerant participants compared with participants with normal glucose tolerance (NGT). This finding is usually taken as proof of defective compensation, as for any given level of insulin resistance, AIR is lower in hyperglycaemic than in control participants. However, if the lines are parallel, then the increase in AIR for a given increase in insulin resistance is the same in both participant groups. If some compensatory physiological mechanism subtended the observed relationships, then hyperglycaemic participants would have normal AIR when IS is high, but progressively subnormal AIR as IS deteriorates; in other words, the line for the hyperglycaemic group would be flatter than that for the control group. In a recent study involving a relatively large number of patients with type 2 diabetes [9], we were unable to confirm the hyperbolic compensation paradigm as the sole mechanism responsible for the emergence of dysglycaemia. Our data and analyses were compatible with the idea that beta cell adaptation to insulin resistance does not involve all modes of the secretory response to glucose stimulation. To improve our understanding of how beta cell function is modulated by insulin resistance in persons with normal and impaired glucose tolerance, we extended our previous analysis $[9,10]$ to a much larger group of $\sim 1,300$ healthy non-diabetic participants from the European Relationship between Insulin Sensitivity and Cardiovascular disease (RISC) study [11], who underwent a euglycaemichyperinsulinaemic clamp as a direct measure of IS, an OGTT for assessment of beta cell function by mathematical modelling and a glucose bolus to estimate AIR.

\section{Methods}

Study participants RISC is a prospective, observational cohort study whose rationale and methodology have been described previously [11]. In brief, participants were recruited from the local population at 19 centres in 14 countries in Europe, according to the following inclusion criteria: both sexes, age range 30 to 60 years and clinically healthy. Initial exclusion criteria were: (1) treatment for obesity, hypertension, lipid disorders or diabetes, pregnancy and cardiovascular or chronic lung disease; (2) weight change of $\geq 5 \mathrm{~kg}$ in the previous 3 months; (3) cancer (in the last 5 years); and (4) renal failure. Exclusion criteria after screening were: arterial blood pressure $\geq 140 / 90 \mathrm{mmHg}$, fasting plasma glucose $\geq 7.0 \mathrm{mmol} / \mathrm{l}, 2 \mathrm{~h}$ plasma glucose (standard $75 \mathrm{~g}$ OGTT) $\geq 11.0 \mathrm{mmol} / \mathrm{l}$, total serum cholesterol $\geq 7.8 \mathrm{mmol} / \mathrm{l}$, serum triacylglycerol $\geq 4.6 \mathrm{mmol} / 1$ or ECG abnormalities. Baseline examinations began in June 2002 and were completed in November 2005. The present analysis is based on the baseline data of 1,279 participants who satisfied all criteria, were non-diabetic according to OGTT and the 1997 ADA criteria [12], and for whom all variables used for this analysis were available. These participants were classified as having NGT (defined as fasting glucose $<6.1 \mathrm{mmol} / 1$ and $2 \mathrm{~h}$ glucose $<7.8 \mathrm{mmol} / \mathrm{l}$ ) or impaired glucose regulation (IGR), the latter encompassing impaired fasting glycaemia, impaired glucose tolerance and a combination of both.

Local Ethics Committee approval was obtained by each recruiting centre. Volunteers were given detailed written information on the study as well as oral explanations and they all signed a consent form.

OGTT On the first day, in the morning after an overnight fast, a $75 \mathrm{~g}$ OGTT was performed with sampling at baseline and at 30,60, 90 and $120 \mathrm{~min}$ after glucose ingestion for measurement of glucose, insulin and Cpeptide concentrations. 
Glucose clamp On a separate day within 1 to 3 weeks of the OGTT, a euglycaemic-hyperinsulinaemic clamp was performed on all participants. Exogenous insulin was administered as a primed continuous infusion at a rate of 240 pmol min m $^{-1}$ simultaneously with a variable $20 \%$ dextrose infusion adjusted every $5 \mathrm{~min}$ to maintain plasma glucose within $0.8 \mathrm{mmol} / \mathrm{l}( \pm 15 \%)$ of the target glucose level (4.5-5.5 mmol/l). The clamp procedure was standardised across centres with the use of a demonstration video and written operating instructions. The raw data from each clamp study were immediately transferred to the coordinating centre, where they underwent quality control scrutiny according to pre-set criteria. Blood was sampled throughout the clamp for the measurement of glucose. A fasting blood sample and two samples during the last $40 \mathrm{~min}$ of the clamp were taken for measurement of glucose, insulin and C-peptide concentrations.

Glucose bolus At the end of the clamp and while the clamp was still in progress, a glucose bolus $(0.3 \mathrm{mg} / \mathrm{kg}$ body weight) was administered over $1 \mathrm{~min}$ to 904 participants; plasma glucose and C-peptide concentrations were measured at 2, 4, 6 and 8 min after the bolus.

Analytical methods Blood collected during the studies was separated into plasma and serum, divided into aliquots and stored at $-20^{\circ} \mathrm{C}$ for glucose and at $-80^{\circ} \mathrm{C}$ for insulin and $\mathrm{C}$ peptide measurement in the central laboratories. Samples were transported on dry ice at pre-arranged intervals to the laboratories. Plasma glucose was measured by the glucose oxidase technique. Plasma insulin and C-peptide were measured by a two-sited, time-resolved fluoroimmunoassay (AutoDELFIA Insulin kit; Wallac Oy, Turku, Finland) using monoclonal antibodies, with the following assay characteristics: sensitivity 1 to $2 \mathrm{pmol} / \mathrm{l}$ and $5 \mathrm{pmol} / \mathrm{l}$, respectively; within-assay coefficient of variation $5 \%$ for both; between-assay variation $5 \%$ and $3.5 \%$.

Calculations Insulin sensitivity was expressed as the ratio of the $M$ value [13], calculated during the final $40 \mathrm{~min}$ of the $2 \mathrm{~h}$ clamp, to the mean plasma insulin concentration measured during the same interval $(M / I)$. Insulin sensitivity was normalised to fat-free mass (FFM) as measured by bioimpedance (TBF-300 Bioimpedance Balance; Tanita, West Drayton, UK) and expressed in units of $\mu \mathrm{mol} \mathrm{min} \mathrm{mi}^{-1}$ $\left(\mathrm{kg}_{\mathrm{FFM}}{ }^{-1}\right)(\mathrm{nmol} / \mathrm{l})^{-1}$.

The AIR was assessed using C-peptide deconvolution [14]. AIR was calculated as the ratio of the mean incremental insulin secretion (in pmol $\mathrm{min}^{-1} \mathrm{~m}^{-2}$ of body surface area) during the first $8 \mathrm{~min}$ after the glucose bolus to the mean incremental glucose concentration over the same time interval; the units of AIR were thus pmol $\min ^{-1} \mathrm{~m}^{-2}(\mathrm{mmol} / \mathrm{l})^{-1}$. AIR was also calculated as the mean C-peptide concentration during the first $8 \mathrm{~min}$ following the glucose bolus. Baseline glucose values for calculating the increments were those obtained at the end of the clamp.

Beta cell function was assessed from the OGTT using a model describing the relationship between insulin secretion and glucose concentration; this model has been illustrated in detail previously $[15,16]$ and expresses insulin secretion (in pmol $\mathrm{min}^{-1} \mathrm{~m}^{-2}$ ) as the sum of two components. The first component represents the dependence of insulin secretion on absolute glucose concentration at any time point during the OGTT through a dose-response function relating the two variables. The characteristic variable of the dose-response is the mean slope over the observed glucose range, denoted as beta cell glucose sensitivity. The doseresponse is modulated by a potentiation factor, which accounts for the fact that during an acute stimulation, insulin secretion is higher on the descending phase of hyperglycaemia than at the same glucose concentration on the ascending phase. As such, the potentiation factor encompasses several potentiating mechanisms (prolonged exposure to hyperglycaemia, non-glucose substrates, gastro-intestinal hormones, neural modulation). It is set to be a positive function of time and is constrained to average unity during the experiment. In normal individuals, the potentiation factor typically increases from baseline to the end of a $2 \mathrm{~h}$ OGTT [17]. To quantify this excursion, the ratio between the $2 \mathrm{~h}$ and the baseline value was calculated. This ratio is denoted as the 'potentiation ratio'. The second insulin secretion component represents the dependence of insulin secretion on the rate of change of glucose concentration. This component is termed the derivative component and is determined by a single variable, denoted as 'rate sensitivity'. Rate sensitivity is related to early insulin release [17].

The model variables were estimated from glucose and Cpeptide concentrations by regularised least-squares, as previously described $[15,16]$. Regularisation involves the choice of smoothing factors, which were selected to obtain glucose and C-peptide model residuals with standard deviations close to the expected measurement error $(\sim 1 \%$ for glucose, $\sim 4 \%$ for C-peptide). Insulin secretion rates were calculated from the model every $5 \mathrm{~min}$. The integral of insulin secretion during the 2-h OGTT was denoted as 'total insulin output'.

Statistical methods Data are expressed as mean \pm SD or median (interquartile range) for non-normally distributed variables. Group differences were tested by the MannWhitney $U$ test (for continuous variables) or the $\chi^{2}$ test (for categorical variables), at a significance level of 0.05 . Univariate associations were tested with the Spearman correlation coefficient $(\rho)$. Linear regression was performed 
with standard procedures on log-transformed data. Group differences in the relationship between two variables were determined with an analysis of covariance model including the group and the interaction term between the independent variables.

\section{Results}

Of the 1,279 study participants, 117 had impaired glucose tolerance and 39 had impaired fasting glucose; thus, 156 $(12 \%)$ had IGR. IGR participants had a more marked family history of diabetes, were older and heavier, and had higher fasting and $2 \mathrm{~h}$ plasma glucose concentrations (Table 1).

Figure 1 shows the relationship between IS and fasting insulin secretion, and between IS and total insulin output in NGT and IGR participants. In IGR participants, these relationships were shifted significantly upwards as compared with NGT participants $(p<0.0001$ for the group term; $p=\mathrm{NS}$ for interaction). This upward shift can be explained by the relative hyperglycaemia of the IGR group (Table 1). In contrast, IS was not significantly correlated with beta cell glucose sensitivity in NGT or IGR participants, either in the whole group or in any of the centres (Fig. 1, Table 2). Although significant, the correlation between fasting insulin secretion and glucose sensitivity was weak $(\rho=0.1, p<0.001)$.

A differential relation of IS to fasting secretion vs glucose sensitivity was also evident when these variables were calculated without relying on the beta cell model. Figure 2 shows the relationship between the measured glucose concentrations at the different times during the OGTT and the corresponding insulin secretion rates in NGT participants in the top and bottom quartiles of IS (M/I 226 [53] vs $78[25] \mathrm{pmol} \mathrm{min}^{-1}\left[\mathrm{~kg}_{\mathrm{FFM}}\right]^{-1}[\mathrm{nmol} / 1]^{-1}$, respectively, median [interquartile range], $n=281$ participants in each group). The two curves are virtually parallel, with the relationship in the more insulin-resistant participants shifted upwards against that of more insulin-sensitive participants. This clearly indicates that IS influences the fasting

Table 1 Characteristics of the study cohort

\begin{tabular}{llll}
\hline Variable & NGT & IGR & $p$ value \\
\hline Sex (women/men), $n$ & $624 / 499$ & $86 / 70$ & NS \\
Familial diabetes (\%) & 24 & 44 & 0.0001 \\
Age (years) & $43 \pm 8$ & $46 \pm 8$ & 0.0001 \\
BMI (kg/m ${ }^{2}$ ) & $25.2 \pm 3.9$ & $27.4 \pm 4.3$ & 0.0001 \\
Fasting glucose (mmol/l) & $5.0 \pm 0.5$ & $5.5 \pm 0.7$ & 0.0001 \\
2 h glucose (mmol/l) & $5.4 \pm 1.1$ & $8.1 \pm 1.4$ & 0.0001 \\
\hline
\end{tabular}

Values are mean $\pm \mathrm{SD}$
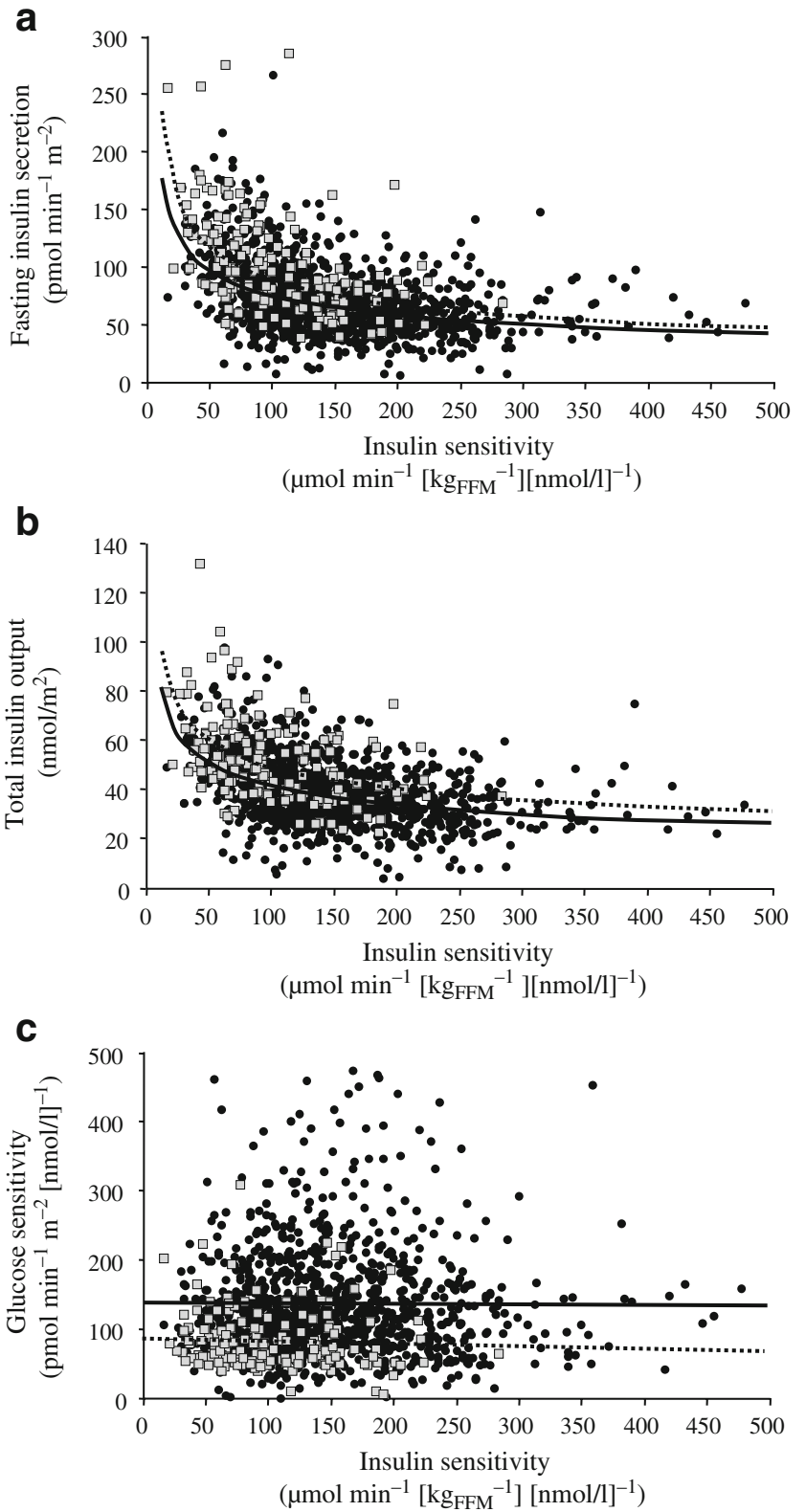

Fig. 1 Reciprocal relationship between IS and fasting insulin secretion rate (a) and total insulin output (b) in NGT (black circles and solid lines) and IGR participants (grey squares and dotted lines). Non-significant relationship between IS and glucose sensitivity (c). The curves are power fits of the data

Table 2 Spearman correlation coefficients $(\rho)$ and partial correlation coefficients $^{\mathrm{a}}$ between IS and beta cell function variables in NGT participants

\begin{tabular}{lrlll}
\hline Variable & $\rho$ & $p$ value & Partial $r$ & $p$ value \\
\hline Fasting insulin secretion & -0.43 & $<0.0001$ & -0.23 & $<0.0001$ \\
Total insulin output & -0.44 & $<0.0001$ & -0.34 & $<0.0001$ \\
Glucose sensitivity & 0.01 & NS & -0.05 & NS \\
Potentiation ratio & 0.16 & $<0.0001$ & 0.05 & NS \\
AIR & -0.15 & $<0.0001$ & -0.11 & $<0.02$ \\
\hline
\end{tabular}

${ }^{\text {a }}$ Partialled with respect to centre, sex, age and BMI 


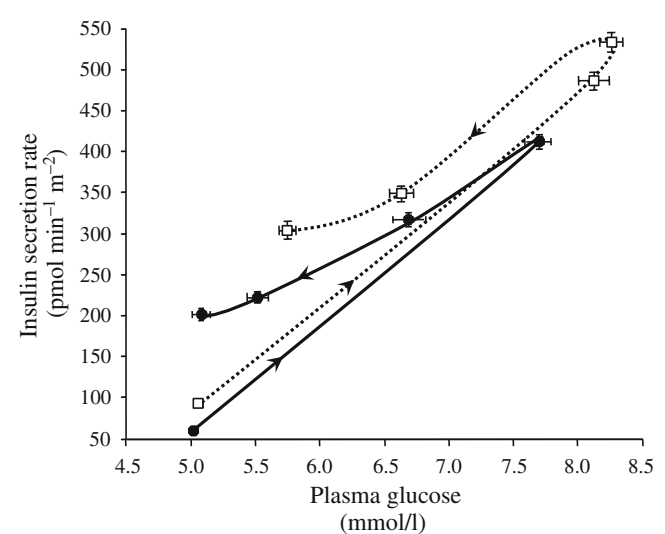

Fig. 2 Plot of insulin secretion rates against the corresponding plasma glucose concentrations during the OGTT in NGT participants in the top quartile (solid line, black circles) and those in the bottom quartile (dotted line, white squares) of IS. Data are mean \pm SEM. Arrows, direction of time during OGTT, from time 0 to $120 \mathrm{~min}$

secretory tone but not the slope of the relationship, i.e. glucose sensitivity.

With the glucose bolus, the incremental glucose area (over the first $8 \mathrm{~min}$ after injection) averaged $6.9 \pm$ $2.0 \mathrm{mmol} / \mathrm{l}$, i.e. a $30 \% \mathrm{CV}$, while the corresponding incremental insulin secretion averaged $3.47 \pm 1.91 \mathrm{nmol} / \mathrm{m}^{2}$, i.e. a $\mathrm{CV}$ of $55 \%$, similar to the $60 \% \mathrm{CV}$ of model-derived glucose sensitivity.

As shown in Table 3, in comparison with NGT participants, IGR participants were less insulin-sensitive and had lower glucose sensitivity ( $42 \%$ loss). Fasting insulin secretion was higher in absolute values and AIR was lower ( $21 \%$ loss), as was potentiation. Each of these group differences retained statistical significance after adjusting for centre, sex, age and BMI; in fact, upon adjustment, rate sensitivity was also significantly $(p=0.036)$ lower in IGR than in NGT participants.

As expected [4], a significant inverse correlation was also observed between IS and AIR, regardless of whether the latter was expressed as an incremental secretion corrected for the glucose rise or, more traditionally, as the C-peptide concentration response. By fitting reciprocal functions separately to the data of NGT and IGR partic- ipants, the resulting curves were compatible with a leftward shift (i.e. different intercept but similar slope) in IGR vs NGT participants with either dataset (Fig. 3).

AIR was not only related to IS, but also to other factors. In a multiple regression model including centre, sex, age, BMI, fasting insulin secretion, fasting glucose and glucose tolerance (NGT/IGR) as independent variables, IS was not an independent predictor of AIR (Table 4). Notably, fasting insulin secretion was the strongest (positive) predictor; if removed, IS became strongly significant.

We next assessed the independent impact of IS and beta cell function on plasma glucose concentrations by setting up multiple regression models with the plasma glucose level at each OGTT time-point as the dependent variable, and IS and all beta cell function variables as independent variables (and further controlling for centre, sex, age and BMI). As plotted in Fig. 4 (in metrics of partial correlation coefficients), the contribution of glucose sensitivity and rate sensitivity rose quickly during the OGTT, the latter peaking and declining earlier than the former. Insulin sensitivity rose gradually to level off during the last $30 \mathrm{~min}$ of the test, whereas potentiation only emerged in the second half of the test. When using the glucose area under the OGTT curve (with a total variance of $41 \%$ explained by the full model), the rank order of predictors was glucose sensitivity $(36 \%$ of explained variance), IS (17\%), rate sensitivity (14\%) and potentiation $(5 \%)$.

\section{Discussion}

Key features of the present study cohort, such as its large size, the selection and characterisation of the participants, the variety (OGTT, clamp, glucose bolus) and standardisation of the methods, provided a unique opportunity to systematically explore the relationship between IS and beta cell function. The main findings were: (1) the inverse relationship between beta cell function and IS involves several beta cell function variables, in particular fasting insulin secretion and AIR, but is not universal; (2) beta cell glucose sensitivity is largely independent of IS, but (3) is a
Table 3 Insulin sensitivity and beta cell function variables in participants with NGT or IGR

Values are median (interquartile range)

\begin{tabular}{|c|c|c|c|}
\hline Variable & NGT & IGR & $p$ value \\
\hline IS $\left(\mu \mathrm{mol} \min ^{-1}\left[\mathrm{~kg}_{\mathrm{FFM}}\right]^{-1}[\mathrm{nmol} / 1]^{-1}\right)$ & $133(86)$ & $92(75)$ & $<0.0001$ \\
\hline Total insulin output $\left(\mathrm{nmol} \mathrm{m} \mathrm{m}^{-2}\right)$ & $38(16)$ & $48(21)$ & $<0.0001$ \\
\hline Fasting insulin secretion (pmol $\min ^{-1} \mathrm{~m}^{-2}$ ) & $67(35)$ & $89(56)$ & $<0.0001$ \\
\hline Glucose sensitivity (pmol $\min ^{-1} \mathrm{~m}^{-2}[\mathrm{mmol} / 1]^{-1}$ ) & $119(83)$ & $69(46)$ & $<0.0001$ \\
\hline Potentiation ratio (fold) & $1.73(1.28)$ & $1.46(0.82)$ & 0.002 \\
\hline Rate sensitivity $\left(\mathrm{nmol} \mathrm{m} \mathrm{m}^{-2}[\mathrm{mmol} / \mathrm{l}]^{-1}\right)$ & $0.82(1.30)$ & $0.72(0.82)$ & NS \\
\hline $\operatorname{AIR}\left(\mathrm{pmol} \min ^{-1} \mathrm{~m}^{-2}[\mathrm{mmol} / 1]^{-1}\right)$ & $60(45)$ & $47(37)$ & $<0.0001$ \\
\hline
\end{tabular}




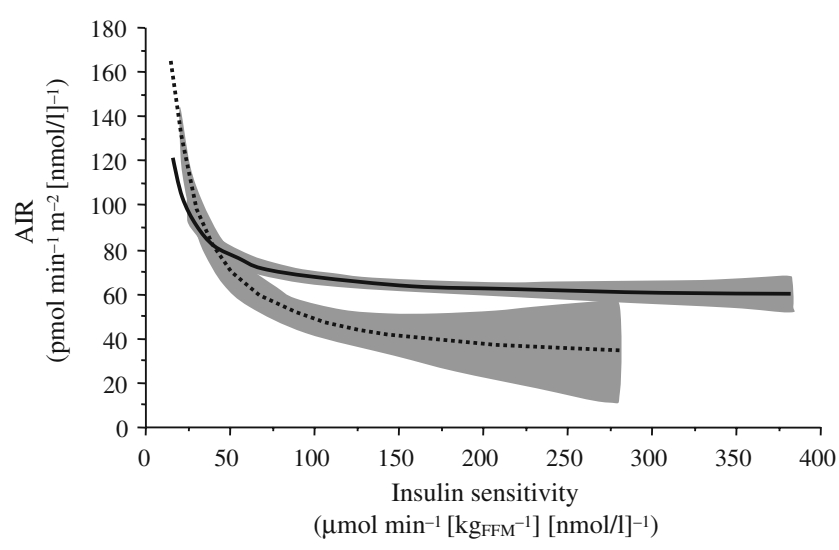

Fig. 3 Curvilinear fit (with 95\% CIs, shaded areas) of the relationship between IS and AIR in NGT (solid line) and IGR (dotted line) participants. The respective equations are: $y=57+1,023 / x, r=0.15$, $p<0.0001$ for NGT; and $y=26+2,159 / x, r=0.46, p<0.0001$ for IGR

key determinant of glucose tolerance; and (4) cross-sectional data show little evidence of defective compensation in IGR states. These conclusions require specification and comment.

First, indices reflecting the secretory tone, such as fasting secretion rate and total insulin output all correlated with IS in a reciprocal fashion and independently of sex, age and obesity (Fig. 1, Table 2). This supports the classical notion that in the presence of insulin resistance the beta cell upregulates its output of insulin. In this restricted sense, beta cell function adapts to insulin action. It remains to be firmly established whether chronic small elevations in plasma glucose concentration or other metabolites and hormones (e.g. NEFA or glucagon-like peptide-1 [7, 18]) are responsible for revving up the secretory tone.

In contrast, dynamic indices of beta cell function, such as glucose sensitivity and potentiation were largely independent of IS (Table 2, Fig. 1). Nevertheless, glucose sensitivity made the largest contribution to overall glucose tolerance (Fig. 4), with additional significant contributions from rate sensitivity and potentiation. Regardless of mathematical modelling, the primary data allow verification of the notion that glucose sensitivity is an inherent feature of beta cell response and one not influenced by IS. Thus, as shown in Fig. 2, the relation of measured secretion rates to

Table 4 Correlates of AIR

\begin{tabular}{lcl}
\hline Variable & Partial $r$ & $p$ value \\
\hline Sex (male) & -0.10 & $<0.03$ \\
Age & -0.16 & $<0.0001$ \\
BMI & -0.04 & NS \\
IGR (vs NGT) & -0.11 & $<0.0001$ \\
Fasting insulin secretion & 0.24 & $<0.0001$ \\
IS & -0.03 & NS \\
Fasting glucose & -0.15 & $<0.0001$ \\
\hline
\end{tabular}

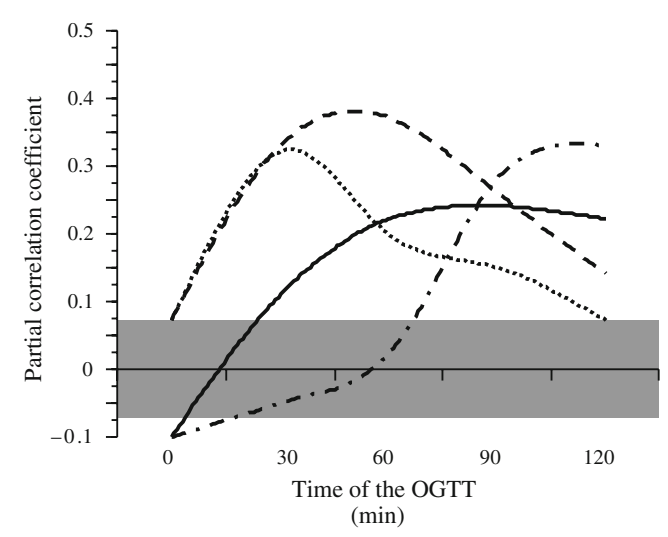

Fig. 4 Partial correlation coefficients (with inverted sign) between plasma glucose concentrations during the OGTT and metabolic variables. The model is: glucose $=(a \times$ centre $)+(b \times \operatorname{sex})+(c \times$ age $)+(d \times \mathrm{BMI})+e \ln [\mathrm{IS}]+f \ln [$ glucose sensitivity $]+g$ [potentiation ratio] $+h$ [rate sensitivity], where $a . . . h$ are regression coefficients. The lines are spline functions connecting the partial correlation coefficients at the five OGTT time-points for IS (solid line), beta cell glucose sensitivity (dashed line), rate sensitivity (dotted line) and potentiation ratio (dashed and dotted line). Grey area, non-significant values for the partial correlation coefficients

the actual glucose concentrations during the OGTT was shifted upward in insulin-resistant vs insulin-sensitive participants with NGT. In other words, insulin-resistant individuals secrete more insulin at each glucose level, but their ability to increase insulin secretion in response to increments in glucose levels during stimulation is not different from that of more insulin-sensitive participants. Thus, adaptation does not extend to glucose sensing. We were unable to determine whether lack of adaptation of glucose sensing is specific to the OGTT. However, it should be noted that studies employing the stepped glucose infusion technique to measure beta cell function (pioneered by Polonsky's group) have yielded results in agreement with our OGTT results, although that point was not made explicit $[19,20]$. In particular, in the study by Jones et al. [19], insulin resistance was characterised by a clear upward shift of the beta cell dose-response, but its slope was not increased, similarly to our Fig. 2. In the report by Gautier et al. [20], the participants at increased risk of diabetes had impaired glucose sensitivity in spite of a similar fasting secretory tone. In addition, in a more recent study employing the graded glucose infusion test in young healthy participants [21], the induction of insulin resistance by administration of nicotinic acid produced an increase in fasting insulin secretion without changing the relationship between insulin secretion and glucose concentration.

In keeping with our assessment of the relative role of IS and beta cell variables in glucose tolerance (Fig. 4), participants with IGR were found to be both insulin- and beta cell glucose-resistant (Table 3) in the face of absolute rates of insulin secretion that were higher than in NGT 
individuals both under fasting conditions and following glucose loading (Fig. 1). Thus, compensation for insulin resistance was active in IGR participants, but glucose regulation was impaired because of reduced beta cell sensing.

In the present study, the glucose bolus was given at the end of the euglycaemic-hyperinsulinaemic clamp, rather than in the fasting state, which is the standard format of the intravenous glucose tolerance test. Although this can be a limitation, in our NGT cohort the average insulin secretion rate during the $8 \mathrm{~min}$ after the bolus was 395 (305) pmol min ${ }^{-1} \mathrm{~m}^{-2}$ (median [interquartile range]), which is consistent with the value of 430 (267) pmol $\mathrm{min}^{-1} \mathrm{~m}^{-2}$ in another group of NGT volunteers receiving the intravenous test in the fasting state [22]. This strongly suggests that euglycaemic pre-insulinisation does not substantially affect AIR. More importantly, our findings fully agree with the notion that this variable is inversely related to IS. Notably, the relationship holds true by using insulin secretion rates or C-peptide levels, but not insulin concentrations, which may overestimate such dependency due to a decrease in insulin clearance in hyperinsulinaemic states [19, 22]. This result also agrees with previous findings [8] indicating a different AIR response in IGR participants compared with NGT participants for the same level of IS (Fig. 3). Thus, our analysis does not contradict the bulk of the literature on this subject based on the hyperbolic model of the crosssectional relationship between IS and secretion [4]. However, as anticipated in the introduction, these data argue against concluding that compensation for insulin resistance is impaired. This would be the case if the relationship between IS and AIR were less steep in IGR than in NGT participants; however, the parallel curves (Fig. 3) indicate an intrinsic beta cell function defect, which is the same at all insulin resistance levels. Notably, these conclusions are not dependent on our non-standard first-phase test; indeed, they would be the same if based on literature data.

The simultaneous assessment of AIR, OGTT-derived variables and IS provides insight into the possible reasons underlying the dependence of AIR on IS and its impairment in glucose-intolerant states. In fact, our results suggest that AIR is a hybrid variable, related to fasting insulin secretion and fasting glucose. Its relation to IS is lost when these variables are accounted for statistically (Table 4). These associations suggest that upregulation of AIR with insulin resistance could reflect a primary dependence of the fasting secretory tone on IS. The conceptual model could be as follows. First, the rapid discharge of insulin that follows a pulse increase in glycaemia is at least in part dependent on the pre-existing magnitude of the immediately releasable pool. Second, the size of this pool depends on the balance between exocytosis and refilling from precursor pools. Third, an increased fasting secretion translates into an increased size of the pool (by refilling), while an increase in ambient glucose levels tends to decrease the pool by stimulation of exocytosis. And fourth, AIR is also dependent on factors other than the pool size, possibly related to glucose sensing (e.g. calcium signalling).

The converging OGTT and glucose bolus results of our cross-sectional study indicate, by extrapolation, the mechanism for the development of hyperglycaemia in IGR states. Individuals can compensate for insulin resistance by increasing their fasting secretory tone or set-point, while their beta cell glucose sensing capacity maintains the value typical of the given individual, which is, to a large extent, independent of the ability to regulate the fasting secretory tone. Thus, as insulin resistance progresses, fasting insulin secretion increases and consequently total insulin secretion is also augmented. However, if glucose sensitivity is impaired, total insulin secretion is not sufficient to cope with the increased insulin demand and hyperglycaemia ensues. This results from the coexistence of insulin resistance with an intrinsic defect in beta cell glucose sensing on a background of normal coupling between IS and the fasting secretory tone [23]. Clearly, deterioration of the glucose sensing defect over time is likely to be a further contributor to hyperglycaemia [10], possibly sustained by glucotoxicity. For this reason, longitudinal studies showing progressive loss of beta cell function in relation to IS in participants who develop hyperglycaemia [24] do not necessarily indicate defective compensation, as progressive beta cell dysfunction alone is a sufficient explanation.

Although our study clearly demonstrates that tonic (i.e. the fasting secretory tone) and phasic (e.g. glucose sensitivity) properties of beta cell function, as determined by OGTT, are substantially independent of one another and have a different role in glucose intolerance, it cannot clarify the underlying cellular mechanisms. In particular, our data do not address the question of whether the changes observed in IGR participants reflect proportionate differences in beta cell mass or function alone (or a mixture of the two).

In conclusion, we have shown that in non-diabetic individuals insulin resistance is associated with an increase of specific variables of the insulin secretory response to glucose challenges. In particular, the adaptation to insulin resistance involves the fasting secretory tone, but not glucose sensitivity. On the other hand, beta cell glucose sensitivity plays an essential role in determining glucose levels during an OGTT. In states of reduced glucose tolerance, this function is markedly impaired, while there is little evidence of a defective relationship with IS. Therefore, our results suggest that hyperglycaemia is the consequence of intrinsic beta cell function deficiency rather than of a defect in the mechanisms of compensation for insulin resistance. This conclusion is based on cross- 
sectional data; future prospective analyses of the RISC cohort may further clarify the role of beta cell dysfunction in the development of hyperglycaemia.

Acknowledgements The RISC Study was supported by EU grant QLG1-CT-2001-01252, with additional support from AstraZeneca (Sweden). The EGIR group activities are supported by an unrestricted research grant from Merck Serono, France. For details of the RISC study and investigators, see Electronic supplementary material (ESM).

Duality of interest The authors declare that there is no duality of interest associated with this manuscript.

\section{References}

1. Kahn SE, Porte D Jr (2003) The pathophysiology and genetics of type 2 diabetes mellitus. In: Porte D Jr, Sherwin R, Baron A (eds) Ellenberg and Rifkin's diabetes mellitus, 6th edn. McGraw-Hill, New York, pp 331-365

2. DeFronzo RA, Mandarino L, Ferrannini E (2004) Metabolic and molecular pathogenesis of type 2 diabetes mellitus. In: DeFronzo RA, Ferrannini E, Keen H, Zimmet P (eds) International textbook of diabetes mellitus, 3rd edn. Wiley, Chichester

3. Bergman RN, Ader M, Huecking K, van Citters G (2002) Accurate assessment of beta-cell function: the hyperbolic correction. Diabetes 51(Suppl 1):S212-S220

4. Kahn SE (2003) The relative contributions of insulin resistance and beta-cell dysfunction to the pathophysiology of type 2 diabetes. Diabetologia 46:3-19

5. Stumvoll M, Tataranni PA, Stefan N, Vozarova B, Bogardus C (2003) Glucose allostasis. Diabetes 52:903-909

6. Ahrén B, Pacini G (2005) Islet adaptation to insulin resistance: mechanisms and implications for intervention. Diabetes Obes Metab 7:2-8

7. van Citters GW, Kabir M, Kim SP et al (2002) Elevated glucagonlike peptide-1-(7-36)-amide, but not glucose, associated with hyperinsulinemic compensation for fat feeding. J Clin Endocrinol Metab 87:5191-5198

8. Utzschneider KM, Prigeon RL, Carr DB et al (2006) Impact of differences in fasting glucose and glucose tolerance on the hyperbolic relationship between insulin sensitivity and insulin responses. Diabetes Care 29:356-362

9. Ferrannini E, Gastaldelli A, Miyazaki Y, Matsuda M, Mari A, DeFronzo RA (2005) beta-Cell function in subjects spanning the range from normal glucose tolerance to overt diabetes: a new analysis. J Clin Endocrinol Metab 90:493-500

10. Walker M, Mari A, Jayapaul MK, Bennett SM, Ferrannini E (2005) Impaired beta cell glucose sensitivity and whole-body insulin sensitivity as predictors of hyperglycaemia in non-diabetic subjects. Diabetologia 48:2470-2476

11. Hills SA, Balkau B, Coppack SW et al (2004) The EGIR-RISC Study (The European group for the study of insulin resistance: relationship between insulin sensitivity and cardiovascular disease risk): I. Methodology and objectives. Diabetologia 47:566-570

12. No authors listed (1997) Report of the Expert Committee on the Diagnosis and Classification of Diabetes Mellitus. Diabetes Care 20:1183-1197

13. DeFronzo RA, Tobin JD, Andres R (1979) Glucose clamp technique: a method for quantifying insulin secretion and resistance. Am J Physiol 237:E214-E223

14. van Cauter E, Mestrez F, Sturis J, Polonsky KS (1992) Estimation of insulin secretion rates from C-peptide levels. Comparison of individual and standard kinetic parameters for C-peptide clearance. Diabetes 41:368-377

15. Mari A, Tura A, Gastaldelli A, Ferrannini E (2002) Assessing insulin secretion by modeling in multiple-meal tests: role of potentiation. Diabetes 51(Suppl 1):S221-S226

16. Mari A, Schmitz O, Gastaldelli A, Oestergaard T, Nyholm B, Ferrannini E (2002) Meal and oral glucose tests for the assessment of $\beta$-cell function: modeling analysis in normal subjects. Am J Physiol Endocrinol Metab 283:E1159-E1166

17. Mari A, Ferrannini E (2008) Beta-cell function assessment from modelling of oral tests: an effective approach. Diabetes Obesity and Metabolism 10(Suppl 4):77-87

18. Kim SP, Catalano KJ, Hsu IR, Chiu JD, Richey JM, Bergman RN (2007) Nocturnal free fatty acids are uniquely elevated in the longitudinal development of diet-induced insulin resistance and hyperinsulinemia. Am J Physiol Endocrinol Metab 292:E1590-E1598

19. Jones CN, Pei D, Staris P, Polonsky KS, Chen YD, Reaven GM (1997) Alterations in the glucose-stimulated insulin secretory doseresponse curve and in insulin clearance in nondiabetic insulinresistant individuals. J Clin Endocrinol Metab 82:1834-1838

20. Gautier JF, Wilson C, Weyer C et al (2001) Low acute insulin secretory responses in adult offspring of people with early onset type 2 diabetes. Diabetes 50:1828-1833

21. Chang AM, Smith MJ, Galecki AT, Bloem CJ, Halter JB (2006) Impaired beta-cell function in human aging: response to nicotinic acid-induced insulin resistance. J Clin Endocrinol Metab 91:3303-3309

22. Mari A, Tura A, Pacini G, Kautzky-Willer A, Ferrannini E (2008) Relationships between insulin secretion after intravenous and oral glucose administration in subjects with glucose tolerance ranging from normal to overt diabetes. Diabet Med 25:671-677

23. Cerasi E (1995) Insulin deficiency and insulin resistance in the pathogenesis of NIDDM: is a divorce possible? Diabetologia 38:992-997

24. Weyer C, Bogardus C, Mott DM, Pratley RE (1999) The natural history of insulin secretory dysfunction and insulin resistance in the pathogenesis of type 2 diabetes mellitus. J Clin Invest 104:787-794 